\title{
Metabolically healthy obesity: Is it really benign?
}

\author{
Nikolaos Kourris, Konstantinos Tziomalos
}

First Propedeutic Department of Internal Medicine, Medical School, Aristotle University of Thessaloniki, AHEPA Hospital, Thessaloniki, Greece

\begin{abstract}
Recently, a subgroup of patients with obesity but without cardiometabolic abnormalities has attracted considerable attention and has been characterized as metabolically healthy obese (MHO) patients. $\mathrm{MHO}$ is quite prevalent among patients with obesity. Even though these subjects have less pronounced metabolic abnormalities compared with patients with metabolically unhealthy obesity (MUO), they are at increased risk for progressing to $\mathrm{MUO}$ and for developing cardiovascular disease. Accordingly, diet, exercise and appropriate pharmacotherapy should be recommended to patients with MHO as strongly as in those with MUO.
\end{abstract}

KEY WORDS: Obesity, metabolically healthy obesity, cardiovascular risk, metabolic syndrome

Undoubtedly, obesity represents one of the most important public health problems ${ }^{1}$. In the last decades, a steady increase of its prevalence has been observed and it is expected that by 2030 over a billion people are going to suffer from its consequences ${ }^{1}$. Indeed, obesity is a major risk factor for type 2 diabetes mellitus (T2DM), non-alcoholic fatty liver disease (NAFLD), cardiovascular disease (CVD) and various types of cancer ${ }^{2}$. However, over the past years, a subgroup of patients with obesity that has been coined metabolically healthy obese (MHO) has attracted the attention of the medical community ${ }^{3}$. Despite the interest on this subset of patients with obesity, there is still no clear, uniform definition of $\mathrm{MHO}^{3}$. A frequently used definition of $\mathrm{MHO}$ is obesity without any of the

\section{Corresponding author:}

Konstantinos Tziomalos, MD, PhD,

Associate Professor of Internal Medicine

First Propedeutic Department of Internal Medicine,

AHEPA Hospital

1 Stilponos Kyriakidi Street, Thessaloniki, 54636, Greece

Tel.: +30 2310994621

E-mail:ktziomalos@yahoo.com diagnostic criteria of the metabolic syndrome (MetS), i.e. high blood pressure, high levels of triglycerides, low levels of high-density lipoprotein cholesterol (HDL-C) and high levels of glucose ${ }^{3}$. The absence of insulin resistance and normal high-sensitivity C-reactive protein (hsCRP) levels has also been used to distinguish between $\mathrm{MHO}$ and metabolically unhealthy obese (MUO) individuals ${ }^{3}$. The exact prevalence of $\mathrm{MHO}$ is also unclear and varies between studies, ranging between 10 and $40 \%$ of the patients with obesity ${ }^{4}$.

Subjects with $\mathrm{MHO}$ display a more favourable metabolic profile than patients with $\mathrm{MUO}^{5}$. Indeed, the former are more insulin sensitive and have lower glucose and insulin levels ${ }^{5}$. Furthermore, some studies reported lower $\mathrm{HbA}_{1 \mathrm{c}}$ levels in $\mathrm{MHO}$ subjects ${ }^{5}$. Regarding the lipid profile, patients with $\mathrm{MHO}$ have lower triglyceride, oxidized low-density lipoprotein cholesterol (LDL-C) and free fatty acid levels than patients with MUO, whereas the concentration of $\mathrm{HDL}-\mathrm{C}$ is higher in the former ${ }^{5}$. Waist circumference is also smaller in $\mathrm{MHO}$ individuals ${ }^{5}$. Transaminase and gamma-

Submission: 07.03.2021, Acceptance: 17.04.2021 
glutamyltransferase levels, i.e. markers of NAFLD, are also lower in these patients than in subjects with $\mathrm{MUO}^{5}$. Levels of several markers of subclinical inflammation, including hsCRP, interleukin-6, tumor necrosis factor a and white blood cell count are also lower in patients with $\mathrm{MHO}^{5,6}$. In addition, levels of adiponectin, an insulin-sensitizing adipokine, are elevated in these patients whereas leptin levels are lower ${ }^{5,7}$.

Several factors appear to protect against the development of adverse metabolic characteristics in patients with obesity, giving rise to the MHO phenotype. Increased birth weight and early weight gain appear to predispose to the MHO profile ${ }^{8}$. Early onset of obesity also appears to be related to $\mathrm{MHO}$, particularly in women ${ }^{8}$. Female gender also appears to confer protection against development of $\mathrm{MUO}^{4,8}$. Younger age, African ancestry and a healthier lifestyle, including more vigorous physical activity, no smoking, lower consumption of red meat and higher ratio of monounsaturated to saturated fatty acids in the diet also appear to be associated with $\mathrm{MHO}$ in patients with obesity ${ }^{3}$. In contrast, greater mass of visceral and ectopic fat as well as hypertrophy, dysfunction and inflammation of the adipose tissue contribute to the development of MUO, by affecting the balance between insulin-sensitizing adipokines and adipokines promoting insulin resistance ${ }^{3}$. It has also been suggested that $\mathrm{MHO}$ subjects have greater ability to expand the subcutaneous adipose tissue, which is insulin-sensitive ${ }^{3}$. Finally, genetic factors also appear to be implicated in the favourable metabolic profile of $\mathrm{MHO}$, including the fatty mass and obesity (FTO) gene and loci near the insulin receptor substrate 1 and the sprouty homolog 2 genes ${ }^{9}$.

A critical question pertaining to $\mathrm{MHO}$ is whether these patients are at risk for developing MUO. In a meta-analysis of 12 cohort studies ( $n=32,117), 49 \%$ of subjects with MHO developed at least 1 metabolic disorder within 10 years. Moreover, subjects with $\mathrm{MHO}$ had $80 \%$ greater risk for developing a metabolic disorder than metabolically healthy subjects with a normal body mass index (BMI $)^{4}$. In another meta-analysis of 14 cohort studies ( $n=140,845)$, patients with $\mathrm{MHO}$ had 4.1 times greater risk for developing T2DM than metabolically healthy subjects with normal $\mathrm{BMI}^{10}$.

Even though some patients with $\mathrm{MHO}$ will not develop metabolic disorders, $\mathrm{MHO}$ per se appears to be associated with increased cardiovascular risk. In a systematic review of 6 studies, patients with MHO (without MetS or insulin resistance) had greater carotid intima-media thickness and more severe coronary artery calcification than metabolically healthy subjects with normal $\mathrm{BMI}^{11}$. In a meta-analysis of 22 prospective studies ( $n=584,799$ ), patients with obesity without MetS had 45\% higher risk for cardiovascular events than subjects without MetS but with normal $\mathrm{BMI}^{12}$. In addition, in another meta-analysis of 97 cohort studies ( $n=1,800,000$ ), only $50 \%$ of the excess risk for coronary heart disease in patients with obesity was due to the presence of T2DM, dyslipidemia or hypertension, suggesting that $\mathrm{MHO}$ is also associated with increased cardiovascular risk ${ }^{13}$.

Given the high risk of patients with $\mathrm{MHO}$ to progress to MUO and to experience cardiovascular events, aggressive and multifactorial management aiming at weight loss and at control of metabolic comorbidities is essential. However, in a meta-analysis of 12 studies ( $n=1,827$ ), diet induced smaller reductions in body weight, blood pressure and trigluceride levels in patients with $\mathrm{MHO}$ than in those with MUO and also had no effect on insulin resistance and on glucose and hsCRP levels ${ }^{14}$.

In conclusion, $\mathrm{MHO}$ is quite prevalent among patients with obesity. Even though these subjects have less pronounced metabolic abnormalities compared with patients with MUO, they are at increased risk for progressing to MUO and for developing CVD. Accordingly, diet, exercise and appropriate pharmacotherapy should be recommended to patients with $\mathrm{MHO}$ as strongly as in those with MUO.

\section{Conflict of interest}

None to declare.

\title{
ПЕРІ^НЧН
}

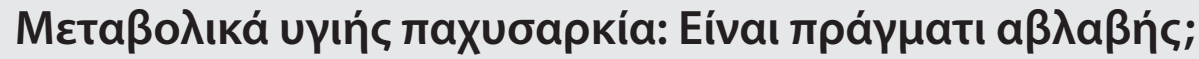

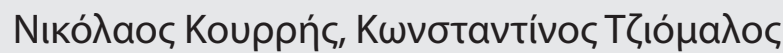

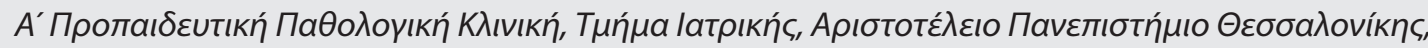

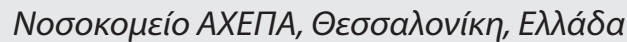

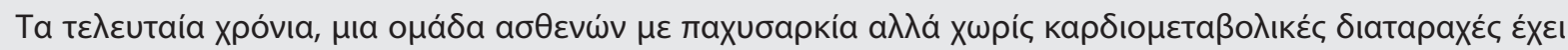

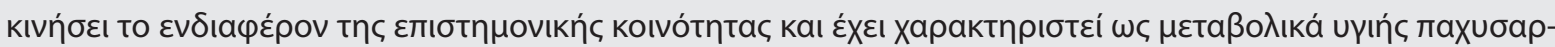

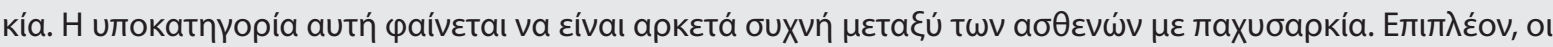




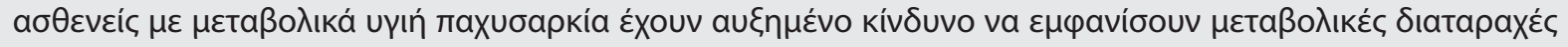

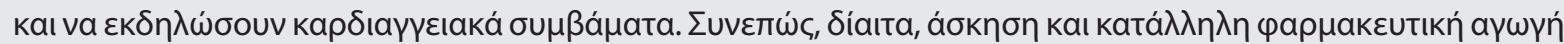

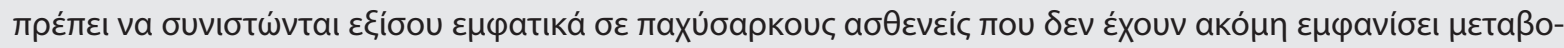

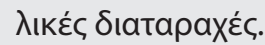

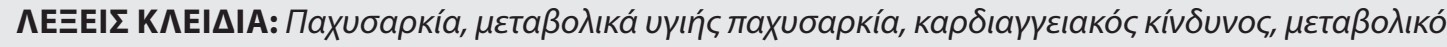

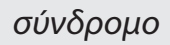

\section{REFERENCES}

1. Ng M, Fleming T, Robinson M, Thomson B, Graetz N, Margono $C$, et al. Global, regional, and national prevalence of overweight and obesity in children and adults during 1980-2013: A systematic analysis for the Global Burden of Disease Study 2013. Lancet. 2014 Aug 30;384(9945):766-81.

2. Global BMI Mortality Collaboration, Di Angelantonio E, Bhupathiraju ShN, Wormser D, Gao P, Kaptoge S, Berrington de Gonzalez A, et al. Body-mass index and allcause mortality: individual-participant-data meta-analysis of 239 prospective studies in four continents. Lancet. 2016 Aug 20;388(10046):776-86.

3. Phillips CM. Metabolically healthy obesity across the life course: Epidemiology, determinants, and implications. Ann NY Acad Sci. 2017 Mar;1391(1):85-100.

4. Lin $H$, Zhang $L$, Zheng $R$, Zheng $Y$. The prevalence, metabolic risk and effects of lifestyle intervention for metabolically healthy obesity: a systematic review and meta-analysis: A PRISMA-compliant article. Medicine (Baltimore). 2017 Nov;96(47):e8838.

5. Primeau V, Coderre L, Karelis AD, Brochu M, Lavoie ME, Messier V, et al. Characterizing the profile of obese patients who are metabolically healthy. Int J Obes (Lond). 2011 Jul;35(7):971-81.

6. Karelis AD, Faraj M, Bastard JP, St-Pierre DH, Brochu M, Prud'homme $D$, et al. The metabolically healthy but obese individual presents a favorable inflammation profile. J Clin Endocrinol Metab. 2005 Jul;90(7):4145-50.

7. Aguilar-Salinas CA, García EG, Robles L, Riaño D, RuizGomez DG, García-Ulloa AC, et al. High adiponectin concentrations are associated with the metabolically healthy obese phenotype. J Clin Endocrinol Metab. 2008 Oct;93(10):4075-9.

8. Blüher M. Metabolically healthy obesity. Endocr Rev. 2020 May;41(3)405-20.

9. Loos RJF, Kilpeläinen TO. Genes that make you fat, but keep you healthy. J Intern Med. 2018 Nov;284(5):450-63.

10. Lotta LA, Abbasi A, Sharp SJ, Sahlqvist AS, Waterworth D, Brosnan JM, et al. Definitions of metabolic health and risk of future type 2 diabetes in BMI categories: A systematic review and network meta-analysis. Diabetes Care. 2015 Nov;38(11):2177-87.

11. Roberson LL, Aneni EC, Maziak W, Agatston A, Feldman T, Rouseff M, et al. Beyond BMI:The"Metabolically healthy obese" phenotype \& its association with clinical/subclinical cardiovascular disease and all-cause mortality - a systematic review. BMC Public Health. 2014 Jan;14:14.

12. Eckel N, Meidtner K, Kalle-UhImann T, Stefan N, Schulze MB. Metabolically healthy obesity and cardiovascular events: A systematic review and meta-analysis. Eur J Prev Cardiol. 2016 Jun;23(9):956-66.

13. Global Burden of Metabolic Risk Factors for Chronic Diseases Collaboration (BMI Mediated Effects), Lu Y, Hajifathalian K, Ezzati M, Woodward M, Rimm EB, Danaei G. Metabolic mediators of the effects of body-mass index, overweight, and obesity on coronary heart disease and stroke: a pooled analysis of 97 prospective cohorts with 1.8 million participants. Lancet. 2014 Mar;383(9921):97083.

14. Stelmach-Mardas M, Walkowiak J. Dietary interventions and changes in cardio-metabolic parameters in metabolically healthy obese subjects: A systematic review with meta-analysis. Nutrients. 2016 Jul 28;8(8):455. 